\title{
Some New Fixed Point Theorems in Complex Valued G-Metric Spaces
}

\author{
Ing-Jer Lin, Wei-Shih Du, and Qiao-Feng Zheng \\ Department of Mathematics, National Kaohsiung Normal University, Kaohsiung 82444, Taiwan \\ Correspondence should be addressed to Wei-Shih Du; wsdu@nknucc.nknu.edu.tw
}

Received 11 May 2014; Accepted 14 June 2014; Published 29 June 2014

Academic Editor: Erdal Karapinar

Copyright (C) 2014 Ing-Jer Lin et al. This is an open access article distributed under the Creative Commons Attribution License, which permits unrestricted use, distribution, and reproduction in any medium, provided the original work is properly cited.

Some new fixed point theorems are established in the setting of complex valued $G$-metric spaces. These new results improve and generalize Kang et al.s results, the Banach contraction principle, and some well-known results in the literature.

\section{Introduction and Preliminaries}

It is well known that Banach contraction principle [1] plays an important role in various fields of applied mathematical analysis and scientific applications and has been generalized and improved in many various different directions; see [2-16] and references therein. In 2011, Azam et al. [2] introduced socalled complex valued metric spaces and proved the existence of fixed points under some contraction conditions. In 2006, Mustafa and Sims [3] introduced the concept of $G$-metric spaces to extend and generalize the notion of metric spaces. In 2013, Kang et al. [8] introduced the concept of complex valued $G$-metric spaces to generalize and improve the notion of $G$-metric spaces. In [8], the authors gave a complex valued $G$-metric version of Banach contraction principle.

In what follows we will give some definitions and known results which will be needed in the sequel. Throughout the present paper, the symbols $\mathbb{N}, \mathbb{R}$, and $\mathbb{C}$ are used to denote the sets of positive integers, real numbers, and complex numbers, respectively.

In 2006, Mustafa and Sims [3] introduced a new class of metric spaces called generalized metric spaces or $G$-metric spaces as follows.

Definition 1 (see [3]). Let $X$ be a nonempty set and let $G$ : $X \times X \times X \rightarrow[0, \infty)$ be a function satisfying the following:
(G1) $G(x, y, z)=0$ if $x=y=z$,
(G2) $0<G(x, y, z)$ for all $x, y \in X$ with $x \neq y$,

(G3) $G(x, x, y) \leq G(x, y, z)$ for all $x, y, z \in X$ with $y \neq z$,

(G4) $G(x, y, z)=G(x, z, y)=G(y, z, x)=\cdots$ (symmetry in all three variables),

(G5) $G(x, y, z) \leq G(x, a, a)+G(a, y, z)$ for all $x, y, z, a \in X$ (rectangle inequality).

Then the function $G$ is called a generalized metric or a $G$ metric on $X$ and the pair $(X, G)$ is called a $G$-metric space.

Example 2 (see [3]). Let $(X, d)$ be a usual metric space. Then $\left(X, G_{1}\right)$ and $\left(X, G_{2}\right)$ are all $G$-metric spaces, where

$$
\begin{gathered}
G_{1}(x, y, z)=d(x, y)+d(y, z)+d(z, x), \\
G_{2}(x, y, z)=\max \{d(x, y), d(y, z), d(z, x)\}
\end{gathered}
$$

for all $x, y, z \in X$.

For any $z_{1}, z_{2} \in \mathbb{C}$, we can define a partial order $\leqslant$ on $\mathbb{C}$ as follows:

$$
z_{1} \lesssim z_{2} \Longleftrightarrow \operatorname{Re}\left(z_{1}\right) \leqq \operatorname{Re}\left(z_{2}\right), \quad \operatorname{Im}\left(z_{1}\right) \leqq \operatorname{Im}\left(z_{2}\right) .
$$

So, it is easy to see that $z_{1} \lesssim z_{2}$ holds if one of the following conditions is satisfied:

(C1) $\operatorname{Re}\left(z_{1}\right)=\operatorname{Re}\left(z_{2}\right)$ and $\operatorname{Im}\left(z_{1}\right)=\operatorname{Im}\left(z_{2}\right)$,

(C2) $\operatorname{Re}\left(z_{1}\right)<\operatorname{Re}\left(z_{2}\right)$ and $\operatorname{Im}\left(z_{1}\right)=\operatorname{Im}\left(z_{2}\right)$, 
(C3) $\operatorname{Re}\left(z_{1}\right)=\operatorname{Re}\left(z_{2}\right)$ and $\operatorname{Im}\left(z_{1}\right)<\operatorname{Im}\left(z_{2}\right)$,

(C4) $\operatorname{Re}\left(z_{1}\right)<\operatorname{Re}\left(z_{2}\right)$ and $\operatorname{Im}\left(z_{1}\right)<\operatorname{Im}\left(z_{2}\right)$.

In particular, we will write $z_{1} \preccurlyeq z_{2}$ if $z_{1} \neq z_{2}$ and one of (C2), (C3), and (C4) is satisfied and we will write $z_{1} \prec z_{2}$ if only (C4) is satisfied.

Remark 3. It is obvious that the following statements hold.

(1) If $0 \preceq z_{1} \preccurlyeq z_{2}$, then $\left|z_{1}\right|<\left|z_{2}\right|$.

(2) If $z_{1} \preceq z_{2}$ and $z_{2} \prec z_{3}$, then $z_{1} \prec z_{3}$.

The idea of complex metric space was initialed by Azam et al. [2].

Definition 4. Let $X$ be a nonempty set. Suppose that the mapping $d: X \times X \rightarrow \mathbb{C}$ satisfies

(C1) $0 \preceq d(x, y)$ for all $x, y \in X$ and $d(x, y)=0$ if and only if $x=y$,

(C2) $d(x, y)=d(y, x)$ for all $x, y \in X$,

(C3) $d(x, y) \preceq d(x, z)+d(z, y)$ for all $x, y, z \in X$.

Then $d$ is called a complex valued metric on $X$ and the pair $(X, d)$ is called a complex valued metric space.

Example 5 (see [6, Example 2]). Let $X=X_{1} \cup X_{2}$ where

$$
\begin{aligned}
& X_{1}=\{z \in \mathbb{C}: \operatorname{Re}(z) \geq 0, \operatorname{Im}(z)=0\}, \\
& X_{2}=\{z \in \mathbb{C}: \operatorname{Re}(z)=0, \operatorname{Im}(z) \geq 0\} .
\end{aligned}
$$

Define $d: X \times X \rightarrow \mathbb{C}$ as follows:

$$
d\left(z_{1}, z_{2}\right)=\left\{\begin{array}{r}
\frac{2}{3}\left|x_{1}-x_{2}\right|+\frac{i}{2}\left|x_{1}-x_{2}\right|, \\
\text { if } z_{1}, z_{2} \in X_{1}, \\
\frac{1}{2}\left|y_{1}-y_{2}\right|+\frac{i}{3}\left|y_{1}-y_{2}\right|, \\
\text { if } z_{1}, z_{2} \in X_{2}, \\
\left(\frac{2}{3} x_{1}+\frac{1}{2} y_{2}\right)+i\left(\frac{1}{2} x_{1}+\frac{1}{3} y_{2}\right), \\
\text { if } z_{1} \in X_{1}, \quad z_{2} \in X_{2}, \\
\left(\frac{2}{2} y_{1}+\frac{2}{3} x_{2}\right)+i\left(\frac{1}{3} y_{1}+\frac{1}{2} x_{2}\right), \\
\text { if } z_{1} \in X_{2}, \quad z_{2} \in X_{1},
\end{array}\right.
$$

where $z_{1}=x_{1}+i y_{1}, z_{2}=x_{2}+i y_{2} \in X$. Then $(X, d)$ is a complete complex valued metric space.

The notion of complex valued $G$-metric space was introduced by Kang et al. [8] to generalize the notion of complex valued metric space and $G$-metric space as follows.

Definition 6 (see [8]). Let $X$ be a nonempty set and let $G_{c}$ : $X \times X \times X \rightarrow \mathbb{C}$ be a function satisfying the following:

(CG1) $G_{c}(x, y, z)=0$ if $x=y=z$,

(CG2) $0 \precsim G_{c}(x, x, y)$ for all $x, y \in X$ with $x \neq y$,
(CG3) $G_{c}(x, x, y) \preceq G_{c}(x, y, z)$ for all $x, y, z \in X$ with $y \neq z$,

(CG4) $G_{c}(x, y, z)=G_{c}(x, z, y)=G_{c}(y, z, x)=\cdots$ (symmetry in all three variables),

(CG5) $G_{c}(x, y, z) \preceq G_{c}(x, a, a)+G_{c}(a, y, z)$ for all $x, y, z, a \in$ $X$ (rectangle inequality).

Then the function $G_{c}$ is called a complex valued generalized metric or a complex valued $G$-metric on $X$. We call the pair $\left(X, G_{c}\right)$ a complex valued $G$-metric space.

Remark 7. In fact, condition (CG2) defined in [8] was stated as follows:

(CG2) $0 \prec G_{c}(x, x, y)$ for all $x, y \in X$ with $x \neq y$.

In this paper, we use the weak version of (CG2) as in Definition 6.

Example 8. Let $X=\mathbb{C}$ and $G_{c}: X \times X \times X \rightarrow \mathbb{C}$ be defined by

$$
\begin{aligned}
G_{c}\left(z_{1}, z_{2}, z_{3}\right)= & \left(\left|x_{1}-x_{2}\right|+\left|x_{2}-x_{3}\right|+\left|x_{3}-x_{1}\right|\right) \\
& +i\left(\left|y_{1}-y_{2}\right|+\left|y_{2}-y_{3}\right|+\left|y_{3}-y_{1}\right|\right)
\end{aligned}
$$

where $z_{i}=x_{i}+i y_{i} \in \mathbb{C}$ for any $i \in\{1,2,3\}$. Then $\left(X, G_{c}\right)$ is a complex valued $G$-metric space.

Definition 9. Let $\left(X, G_{c}\right)$ be a complex valued $G$-metric space. A point $v$ in $X$ is a fixed point of a mapping $T: X \rightarrow X$ if $v=T v$. The set of fixed points of $T$ is denoted by $\mathscr{F}(T)$.

Definition 10 (see [8]). Let $\left(X, G_{c}\right)$ be a complex valued $G^{-}$ metric space and let $\left\{x_{n}\right\}$ be a sequence in $X$. We say that $\left\{x_{n}\right\}$ is complex value $G$-convergent to $x \in X$ if, for every $c \in \mathbb{C}$ with $0 \prec c$, there exists $k \in \mathbb{N}$ such that $G_{c}\left(x, x_{n}, x_{m}\right) \prec c$ for all $n, m \geq k$. We refer to $x$ as the limit of the sequence $\left\{x_{n}\right\}$ and we write $x_{n} \rightarrow x$ as $n \rightarrow \infty$.

Definition 11 (see [8]). Let $\left(X, G_{c}\right)$ be a complex valued $G$ metric space.

(i) A sequence $\left\{x_{n}\right\}$ in $X$ is said to be complex valued GCauchy if, for every $c \in \mathbb{C}$ with $0 \prec c$, there exists $k \in \mathbb{N}$ such that $G_{c}\left(x_{n}, x_{m}, x_{l}\right) \prec c$ for all $n, m, l \geq k$.

(ii) $\left(X, G_{c}\right)$ is said to be complete if every complex valued $G$-Cauchy sequence in $X$ is complex valued $G$ convergent in $X$.

Some crucial facts in complex valued $G_{c}$-metric spaces are listed as follows. First, the following proposition follows easily due to (CG5).

Proposition 12 (see [8]). Let $\left(X, G_{c}\right)$ be a complex valued $G^{-}$ metric space. Then, for any $x, y, z \in X$, the following hold:

(1) $G_{c}(x, y, z) \preceq G_{c}(x, x, y)+G_{c}(x, x, z)$,

(2) $G_{c}(x, y, y) \preceq 2 G_{c}(y, x, y)$. 
Proposition 13 (see [8]). Let $(X, G)$ be a complex valued $G^{-}$ metric space. Then, for a sequence $\left\{x_{n}\right\}$ in $X$ and point $x \in X$, the following are equivalent.

(1) $\left\{x_{n}\right\}$ is complex valued G-convergent to $x$.

(2) $\left|G_{c}\left(x_{n}, x_{n}, x\right)\right| \rightarrow 0$ as $n \rightarrow \infty$.

(3) $\left|G_{c}\left(x_{n}, x, x\right)\right| \rightarrow 0$ as $n \rightarrow \infty$.

(4) $\left|G_{c}\left(x_{m}, x_{n}, x\right)\right| \rightarrow 0$ as $m, n \rightarrow \infty$.

Proposition 14 (see [8]). Let $(X, G)$ be a complex valued $G$ metric space and let $\left\{x_{n}\right\}$ be a sequence in $X$. Then $\left\{x_{n}\right\}$ is complex valued G-Cauchy sequence if and only if $\left|G\left(x_{n}, x_{m}, x_{l}\right)\right| \rightarrow$ 0 as $n, m, l \rightarrow \infty$.

Proposition 15 (see [8]). Let $(X, G)$ be a complex valued $G$ metric space. Then the function $G(x, y, z)$ is jointly continuous in all three of its variables.

The main aim of this paper is to establish some new fixed point theorems which extend and generalize Kang et al.s results in [8], the Banach contraction principle, and some well-known results in the literature.

\section{Main Results}

Recall that a function $\varphi:[0, \infty) \rightarrow[0,1)$ is said to be an $\mathscr{M} \mathscr{T}$-function (or $\mathscr{R}$-function) [11-16] if

$$
\limsup _{s \rightarrow t^{+}} \varphi(s)<1 \quad \forall t \in[0, \infty)
$$

It is obvious that if $\varphi:[0, \infty) \rightarrow[0,1)$ is a nondecreasing function or a nonincreasing function, then $\varphi$ is an $\mathscr{M} \mathscr{T}$ function. So the set of $\mathscr{M} \mathscr{T}$-functions is a rich class.

Recently, Du [13] first proved the following characterizations of $\mathscr{M} \mathscr{T}$-functions.

Theorem 16 (see [13]). Let $\varphi:[0, \infty) \rightarrow[0,1)$ be a function. Then the following statements are equivalent.

(a) $\varphi$ is an $\mathscr{M} \mathscr{T}$-function.

(b) For each $t \in[0, \infty)$, there exist $r_{t}^{(1)} \in[0,1)$ and $\varepsilon_{t}^{(1)}>0$ such that $\varphi(s) \leq r_{t}^{(1)}$ for all $s \in\left(t, t+\varepsilon_{t}^{(1)}\right)$.

(c) For each $t \in[0, \infty)$, there exist $r_{t}^{(2)} \in[0,1)$ and $\varepsilon_{t}^{(2)}>0$ such that $\varphi(s) \leq r_{t}^{(2)}$ for all $s \in\left[t, t+\varepsilon_{t}^{(2)}\right]$.

(d) For each $t \in[0, \infty)$, there exist $r_{t}^{(3)} \in[0,1)$ and $\varepsilon_{t}^{(3)}>0$ such that $\varphi(s) \leq r_{t}^{(3)}$ for all $s \in\left(t, t+\varepsilon_{t}^{(3)}\right]$.

(e) For each $t \in[0, \infty)$, there exist $r_{t}^{(4)} \in[0,1)$ and $\varepsilon_{t}^{(4)}>0$ such that $\varphi(s) \leq r_{t}^{(4)}$ for all $s \in\left[t, t+\varepsilon_{t}^{(4)}\right)$.

(f) For any nonincreasing sequence $\left\{x_{n}\right\}_{n \in \mathbb{N}}$ in $[0, \infty)$, we have $0 \leq \sup _{n \in \mathbb{N}} \varphi\left(x_{n}\right)<1$.

(g) $\varphi$ is a function of contractive factor; for any strictly decreasing sequence $\left\{x_{n}\right\}_{n \in \mathbb{N}}$ in $[0, \infty)$, we have $0 \leq$ $\sup _{n \in \mathbb{N}} \varphi\left(x_{n}\right)<1$.
The following new fixed point theorem is one of the main results of this paper. It can be considered as a complex valued $G$-metric version of Banach contraction principle and will generalize and improve [8, Theorem 2.5] and some wellknown results in the literature.

Theorem 17. Let $\left(X, G_{c}\right)$ be a complete complex valued $G$ metric space and let $T: X \rightarrow X$ be a mapping on $X$. Suppose that there exists a $\mathscr{M} \mathscr{T}$-function $\varphi:[0, \infty) \rightarrow[0,1)$ such that

$$
\begin{array}{r}
G_{c}(T x, T y, T z) \precsim \varphi\left(\left|G_{c}(x, y, z)\right|\right) G_{c}(x, y, z) \\
\forall x, y, z \in X .
\end{array}
$$

Then $T$ has a unique fixed point on $X$.

Proof. Let $x_{0} \in X$ be given. Define the sequence $\left\{x_{n}\right\}$ by

$$
x_{n}=T^{n} x_{0}=T x_{n-1} \quad \text { for each } n \in \mathbb{N}
$$

For each $n \in \mathbb{N}$, by (7), we have

$$
G_{c}\left(x_{n}, x_{n+1}, x_{n+1}\right) \precsim \varphi\left(\left|G_{c}\left(x_{n-1}, x_{n}, x_{n}\right)\right|\right) G_{c}\left(x_{n-1}, x_{n}, x_{n}\right)
$$

which implies

$$
\begin{aligned}
& \left|G_{c}\left(x_{n}, x_{n+1}, x_{n+1}\right)\right| \\
& \quad \leq \varphi\left(\left|G_{c}\left(x_{n-1}, x_{n}, x_{n}\right)\right|\right)\left|G_{c}\left(x_{n-1}, x_{n}, x_{n}\right)\right| .
\end{aligned}
$$

Let $\alpha_{n}=\left|G_{c}\left(x_{n-1}, x_{n}, x_{n}\right)\right|$ for $n \in \mathbb{N}$. Then, by (10), we have

$$
\alpha_{n+1} \leq \varphi\left(\alpha_{n}\right) \alpha_{n}<\alpha_{n} \forall n \in \mathbb{N}
$$

So we know that $\left\{\alpha_{n}\right\}$ is a strictly decreasing sequence in $[0, \infty)$. Applying $(\mathrm{g})$ of Theorem 16 , we obtain

$$
0 \leq \sup _{n \in \mathbb{N}} \varphi\left(\alpha_{n}\right)<1
$$

That is,

$$
0 \leq \sup _{n \in \mathbb{N}} \varphi\left(\left|G_{c}\left(x_{n-1}, x_{n}, x_{n}\right)\right|\right)<1 .
$$

Let

$$
\lambda=\sup _{n \in \mathbb{N}} \varphi\left(\left|G_{c}\left(x_{n-1}, x_{n}, x_{n}\right)\right|\right) .
$$

Then $\lambda \in[0,1)$. For each $n \in \mathbb{N}$, by (10) again, we have

$$
\begin{aligned}
& \left|G_{c}\left(x_{n}, x_{n+1}, x_{n+1}\right)\right| \\
& \quad \leq \varphi\left(\left|G_{c}\left(x_{n-1}, x_{n}, x_{n}\right)\right|\right)\left|G_{c}\left(x_{n-1}, x_{n}, x_{n}\right)\right| \\
& \quad \leq \lambda\left|G_{c}\left(x_{n-1}, x_{n}, x_{n}\right)\right| \leq \lambda^{2}\left|G_{c}\left(x_{n-2}, x_{n-1}, x_{n-1}\right)\right| \\
& \quad \leq \cdots \leq \lambda^{n}\left|G_{c}\left(x_{0}, x_{1}, x_{1}\right)\right| .
\end{aligned}
$$


For any $n, m \in \mathbb{N}$ with $m>n$, by the last inequality and repeated use of (CG5), we get

$$
\begin{aligned}
\mid G_{c} & \left(x_{n}, x_{m}, x_{m}\right) \mid \\
\leq & \left|G_{c}\left(x_{n}, x_{n+1}, x_{n+1}\right)\right|+\left|G_{c}\left(x_{n+1}, x_{n+2}, x_{n+2}\right)\right| \\
& \quad+\cdots+\left|G_{c}\left(x_{m-1}, x_{m}, x_{m}\right)\right| \\
\leq & \left(\lambda^{n}+\lambda^{n+1}+\cdots+\lambda^{m-1}\right)\left|G_{c}\left(x_{0}, x_{1}, x_{1}\right)\right| \\
< & \frac{\lambda^{n}}{1-\lambda}\left|G_{c}\left(x_{0}, x_{1}, x_{1}\right)\right|
\end{aligned}
$$

Since $\lambda \in[0,1), \lim _{n \rightarrow \infty}\left(\lambda^{n} /(1-\lambda)\right)\left|G_{c}\left(x_{0}, x_{1}, x_{1}\right)\right|=0$. Hence, by the last inequality, we obtain

$$
\left|G_{c}\left(x_{n}, x_{m}, x_{m}\right)\right| \longrightarrow 0 \text { as } m, n \longrightarrow \infty \text {. }
$$

For any $n, m, l \in \mathbb{N}$, by Proposition 12 , we obtain

$$
G_{c}\left(x_{n}, x_{m}, x_{l}\right) \preceq G_{c}\left(x_{n}, x_{m}, x_{m}\right)+G_{c}\left(x_{l}, x_{m}, x_{m}\right),
$$

which implies

$$
\left|G_{c}\left(x_{n}, x_{m}, x_{l}\right)\right| \leq\left|G_{c}\left(x_{n}, x_{m}, x_{m}\right)\right|+\left|G_{c}\left(x_{l}, x_{m}, x_{m}\right)\right| \text {. }
$$

From (17) and (19), we get $\left|G_{c}\left(x_{n}, x_{m}, x_{l}\right)\right| \rightarrow 0$ as $n, m, l \rightarrow$ $\infty$. Applying Proposition $14,\left\{x_{n}\right\}$ is a complex valued $G$ Cauchy sequence in $\left(X, G_{c}\right)$. By the completeness of $\left(X, G_{c}\right)$, there exists $v \in X$ such that $\left\{x_{n}\right\}$ is complex valued $G$ convergent to $v$.

Next, we prove that $T v=v$. Assume that $T v \neq v$. For each $n \in \mathbb{N}$, by (7), we have

$$
G_{c}\left(x_{n+1}, T v, T v\right) \preceq \varphi\left(\left|G_{c}\left(x_{n}, v, v\right)\right|\right) G_{c}\left(x_{n}, v, v\right)
$$

which deduces

$$
\begin{aligned}
\left|G_{c}\left(x_{n+1}, T v, T v\right)\right| & \leq \varphi\left(\left|G_{c}\left(x_{n}, v, v\right)\right|\right)\left|G_{c}\left(x_{n}, v, v\right)\right| \\
& <\left|G_{c}\left(x_{n}, v, v\right)\right| .
\end{aligned}
$$

Since $x_{n} \rightarrow v$ as $n \rightarrow \infty$ and $G$ is continuous in all three of its variables, from Proposition 15 and by taking limit from both sides of (21), we get

$$
\left|G_{c}(v, T v, T v)\right| \leq\left|G_{c}(v, v, v)\right|=0 .
$$

Since $0 \precsim G_{c}(v, T v, T v)$, by Remark 3, we know

$$
0<\left|G_{c}(v, T v, T v)\right| .
$$

Hence, taking into account (22) and (23), we have

$$
0<\left|G_{c}(v, T v, T v)\right| \leq 0
$$

which is a contradiction. Therefore $T v=v$ or $v \in \mathscr{F}(T)$.

Finally, we want to show the uniqueness of fixed point of $T$ (i.e., $\mathscr{F}(T)$ is a singleton set). We have shown $v \in \mathscr{F}(T)$, so it suffices to show that $\mathscr{F}(T)=\{v\}$. Let $w \in \mathscr{F}(T)$. Suppose $w \neq v$. By (7), we obtain

$$
G_{c}(v, v, w)=G_{c}(T v, T v, T w) \preceq \varphi\left(\left|G_{c}(v, v, w)\right|\right) G_{c}(v, v, w)
$$

which implies

$$
\left|G_{c}(v, v, w)\right| \leq \varphi\left(\left|G_{c}(v, v, w)\right|\right)\left|G_{c}(v, v, w)\right| .
$$

By (26), we have

$$
\left(1-\varphi\left(\left|G_{c}(v, v, w)\right|\right)\right)\left|G_{c}(v, v, w)\right| \leq 0 .
$$

Since $\varphi\left(\left|G_{c}(v, v, w)\right|\right) \in[0,1)$, we have

$$
\left|G_{c}(v, v, w)\right| \leq 0
$$

which deduces

$$
\left|G_{c}(v, v, w)\right|=0
$$

and hence $G_{c}(v, v, w)=0$. This contradicts (CG2). Therefore, it must be $w=v$ and so $\mathscr{F}(T)=\{v\}$. The proof is completed.

Here, we give a simple example illustrating Theorem 17.

Example 18. Let $X=\mathbb{C}$ and $G_{c}: X \times X \times X \rightarrow \mathbb{C}$ be defined by

$$
\begin{aligned}
G_{c}\left(z_{1}, z_{2}, z_{3}\right)= & \left(\left|x_{1}-x_{2}\right|+\left|x_{2}-x_{3}\right|+\left|x_{3}-x_{1}\right|\right) \\
& +i\left(\left|y_{1}-y_{2}\right|+\left|y_{2}-y_{3}\right|+\left|y_{3}-y_{1}\right|\right),
\end{aligned}
$$

where $z_{i}=x_{i}+i y_{i} \in \mathbb{C}$ for any $i \in\{1,2,3\}$. Then $\left(X, G_{c}\right)$ is a complex valued $G$-metric space. Define $T: X \rightarrow X$ and $\varphi:[0, \infty) \rightarrow[0,1)$ by

$$
\begin{gathered}
T z=\frac{1}{10} z \text { for } z \in X, \\
\varphi(t):= \begin{cases}\frac{4}{5}, & \text { if } t=0, \\
\frac{1}{3}, & \text { if } t>0 .\end{cases}
\end{gathered}
$$

Then $\varphi$ is a $\mathscr{M} \mathscr{T}$-function. For any $z_{1}, z_{2}, z_{3} \in \mathbb{C}$, where $z_{i}=$ $x_{i}+i y_{i}$, we have

$$
T z_{i}=\frac{1}{10} z_{i}=\frac{x_{i}}{10}+i \frac{y_{i}}{10} \text { for any } i \in\{1,2,3\} .
$$

By a routine calculation, one can verify that

$$
G_{c}\left(T z_{1}, T z_{2}, T z_{3}\right) \precsim \phi\left(\left|G_{c}\left(z_{1}, z_{2}, z_{3}\right)\right|\right) G_{c}\left(z_{1}, z_{2}, z_{3}\right) .
$$

So all the hypotheses of Theorem 17 are fulfilled. It is therefore possible to apply Theorem 17 to get the fact that $T$ has a unique fixed point on $X$ (precisely speaking, 0 is the unique fixed point of $T$ ). 
The following fixed point theorem established in Gmetric space is immediate from Theorem 17.

Theorem 19. Let $(X, G)$ be a complete $G$-metric space and let $T: X \rightarrow X$ be a mapping on $X$. Suppose that there exists a $\mathscr{M T}$-function $\varphi:[0, \infty) \rightarrow[0,1)$ such that

$$
G(T x, T y, T z) \leq \varphi(G(x, y, z)) G(x, y, z) \quad \forall x, y, z \in X .
$$

Then $T$ has a unique fixed point on $X$.

Since any nondecreasing function or any nonincreasing function $\varphi:[0, \infty) \rightarrow[0,1)$ is an $\mathscr{M} \mathscr{T}$-function, by applying Theorem 17, we have the following results.

Corollary 20. Let $\left(X, G_{c}\right)$ be a complete complex valued $G$ metric space and let $T: X \rightarrow X$ be a mapping on $X$. Suppose that there exists a nondecreasing function $\varphi:[0, \infty) \rightarrow[0,1)$ such that

$$
\begin{array}{r}
G_{c}(T x, T y, T z) \lesssim \varphi\left(\left|G_{c}(x, y, z)\right|\right) G_{c}(x, y, z) \\
\forall x, y, z \in X .
\end{array}
$$

Then $T$ has a unique fixed point on $X$.

Corollary 21. Let $(X, G)$ be a complete $G$-metric space and let $T: X \rightarrow X$ be a mapping on $X$. Suppose that there exists a nondecreasing function $\varphi:[0, \infty) \rightarrow[0,1)$ such that

$$
G(T x, T y, T z) \leq \varphi(G(x, y, z)) G(x, y, z) \quad \forall x, y, z \in X .
$$

Then $T$ has a unique fixed point on $X$.

Corollary 22. Let $\left(X, G_{c}\right)$ be a complete complex valued $G$ metric space and let $T: X \rightarrow X$ be a mapping on $X$. Suppose that there exists a nonincreasing function $\varphi:[0, \infty) \rightarrow[0,1)$ such that

$$
\begin{array}{r}
G_{c}(T x, T y, T z) \lesssim \varphi\left(\left|G_{c}(x, y, z)\right|\right) G_{c}(x, y, z) \\
\forall x, y, z \in X .
\end{array}
$$

Then $T$ has a unique fixed point on $X$.

Corollary 23. Let $(X, G)$ be a complete $G$-metric space and let $T: X \rightarrow X$ be a mapping on $X$. Suppose that there exists a nonincreasing function $\varphi:[0, \infty) \rightarrow[0,1)$ such that

$$
G(T x, T y, T z) \leq \varphi(G(x, y, z)) G(x, y, z) \quad \forall x, y, z \in X .
$$

Then $T$ has a unique fixed point on $X$.

Corollary 24 (see $[8$, Theorem 2.5$])$. Let $\left(X, G_{c}\right)$ be a complete complex valued $G$-metric space and let $T: X \rightarrow X$ be a contraction mapping on $X$; that is,

$$
G_{c}(T x, T y, T z) \lesssim k G_{c}(x, y, z)
$$

for all $x, y, z \in X$, where $k \in[0,1)$. Then $T$ has a unique fixed point.

\section{Conflict of Interests}

The authors declare that there is no conflict of interests regarding the publication of this paper.

\section{Acknowledgment}

Wei-Shih Du was supported by Grant no. NSC 102-2115-M017-001 of the National Science Council of the Republic of China.

\section{References}

[1] S. Banach, "Sur les operations dans les ensembles abstraits et le urs applications aux equations integrales," Fundamenta Mathematicae, vol. 3, no. 1, pp. 133-181, 1922.

[2] A. Azam, B. Fisher, and M. Khan, "Common fixed point theorems in complex valued metric spaces," Numerical Functional Analysis and Optimization, vol. 32, no. 3, pp. 243-253, 2011.

[3] Z. Mustafa and B. Sims, "A new approach to generalized metric spaces," Journal of Nonlinear and Convex Analysis, vol. 7, no. 2, pp. 289-297, 2006.

[4] E. Karapinar and R. P. Agarwal, "Further fixed point results on G-metric spaces," Fixed Point Theory and Applications, vol. 2013, article 154, 2013.

[5] F. Rouzkard and M. Imdad, "Some common fixed point theorems on complex valued metric spaces," Computers and Mathematics with Applications, vol. 64, no. 6, pp. 1866-1874, 2012.

[6] J. Ahmad, A. Azam, and S. Saejung, "Common fixed point results for contractive mappings in complex valued metric spaces," Fixed Point Theory and Applications, vol. 2014, article 67, 2014.

[7] W. Sintunavarat and P. Kumam, "Generalized common fixed point theorems in complex valued metric spaces and applications," Journal of Inequalities and Applications, vol. 2012, article 84, 2012.

[8] S. M. Kang, B. Singh, V. Gupta, and S. Kumar, "Contraction principle in complex valued $G$-metric spaces," International Journal of Mathematical Analysis, vol. 7, no. 49-52, pp. 25492556, 2013.

[9] L. Gajic and M. Stojakovic, "On mappings with $\varphi$-contractive iterate at a point on generalized metric spaces," Fixed Point Theory and Applications, vol. 2014, article 46, 2014.

[10] R. P. Agarwal, H. H. Alsulami, E. Karapnar, and F. Khojasteh, "Remarks on some recent fi xed point results on quaternionvalued metric spaces," Abstract and Applied Analysis, vol. 2014, Article ID 171624, 8 pages, 2014.

[11] W.-S. Du, "Some new results and generalizations in metric fixed point theory," Nonlinear Analysis, vol. 73, no. 5, pp. 1439-1446, 2010.

[12] Z. He, W.-S. Du, and I.-J. Lin, "The existence of fixed points for new nonlinear multivalued maps and their applications," Fixed Point Theory and Applications, vol. 2011, article 84, 2011.

[13] W.-S. Du, "On coincidence point and fixed point theorems for nonlinear multivalued maps," Topology and Its Applications, vol. 159, no. 1, pp. 49-56, 2012.

[14] W.-S. Du, "On Caristi type maps and generalized distances with applications," Abstract and Applied Analysis, vol. 2013, Article ID 407219, 8 pages, 2013. 
[15] W.-S. Du, E. Karapinar, and N. Shahzad, "The study of fixed point theory for various multivalued non-self-maps," Abstract and Applied Analysis, vol. 2013, Article ID 938724, 9 pages, 2013.

[16] W.-S. Du and E. Karapinar, "A note on Caristi-type cyclic maps: related results and applications," Fixed Point Theory and Applications, vol. 2013, article 344, 2013. 


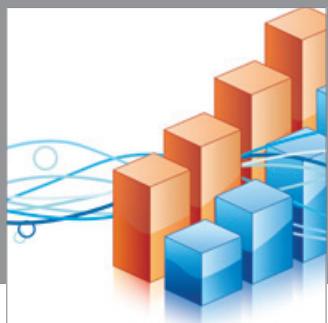

Advances in

Operations Research

mansans

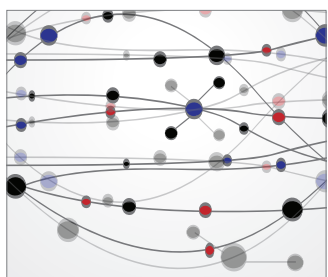

The Scientific World Journal
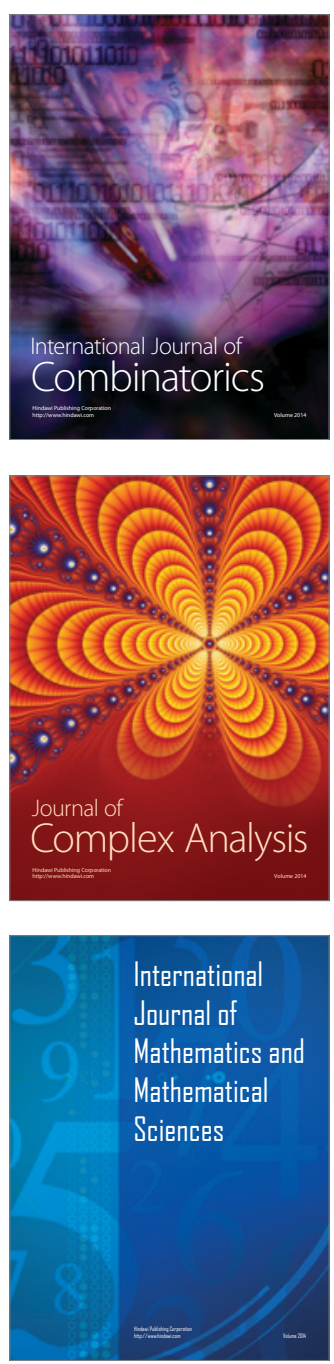
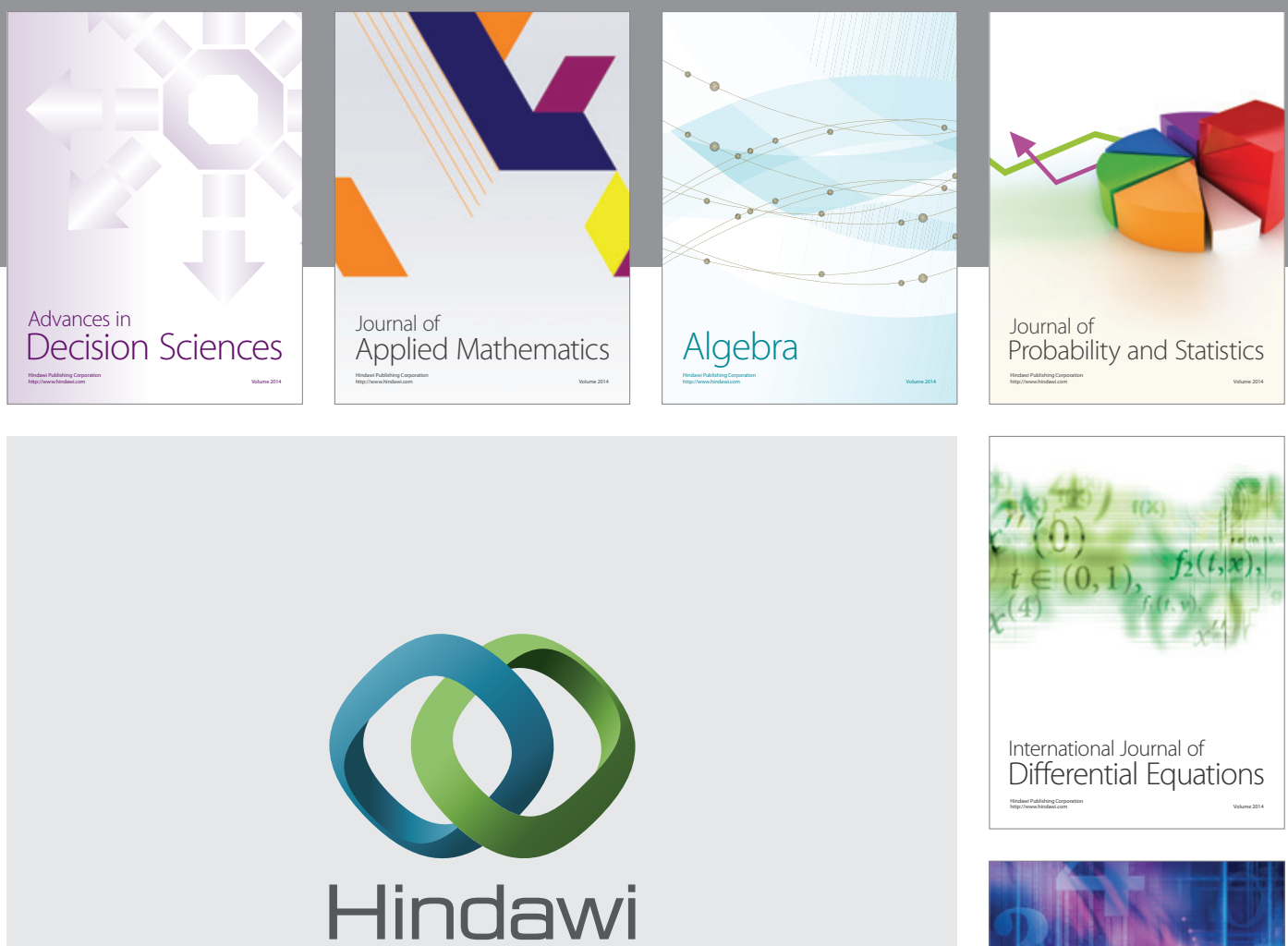

Submit your manuscripts at http://www.hindawi.com
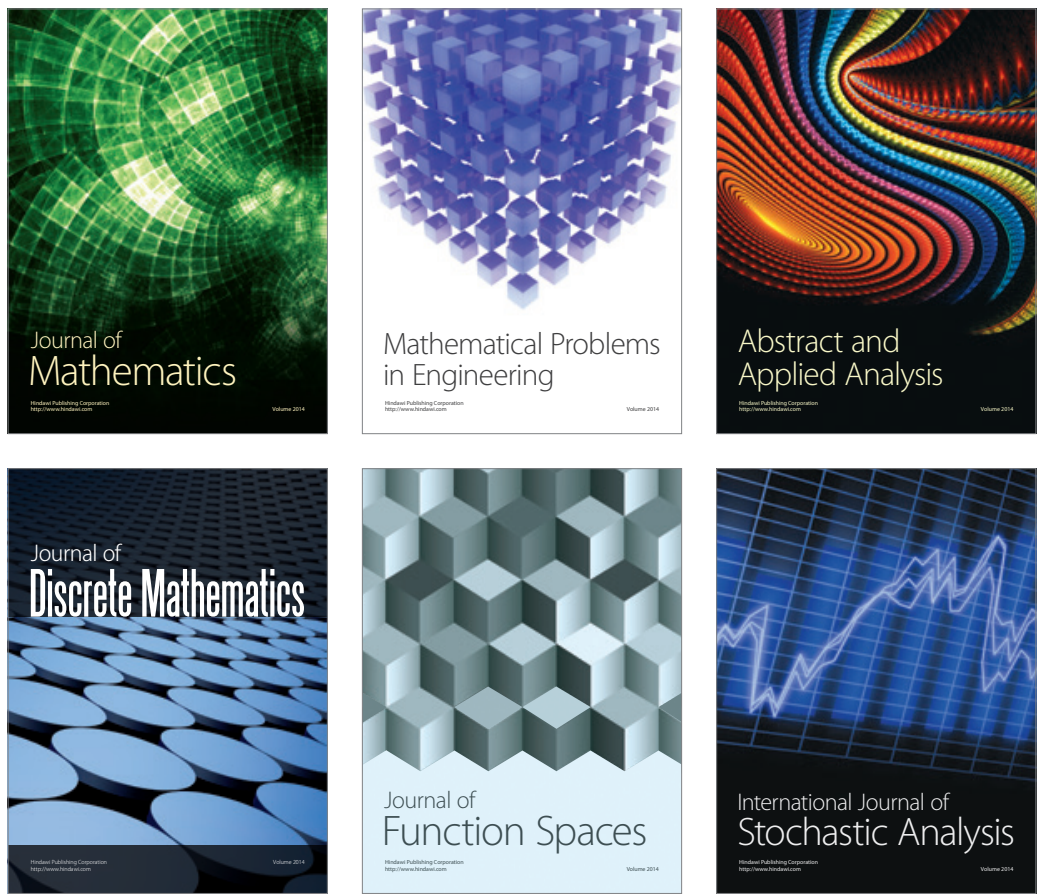

Journal of

Function Spaces

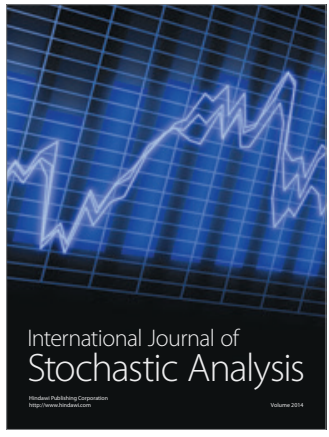

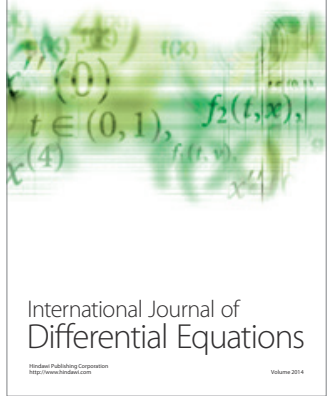
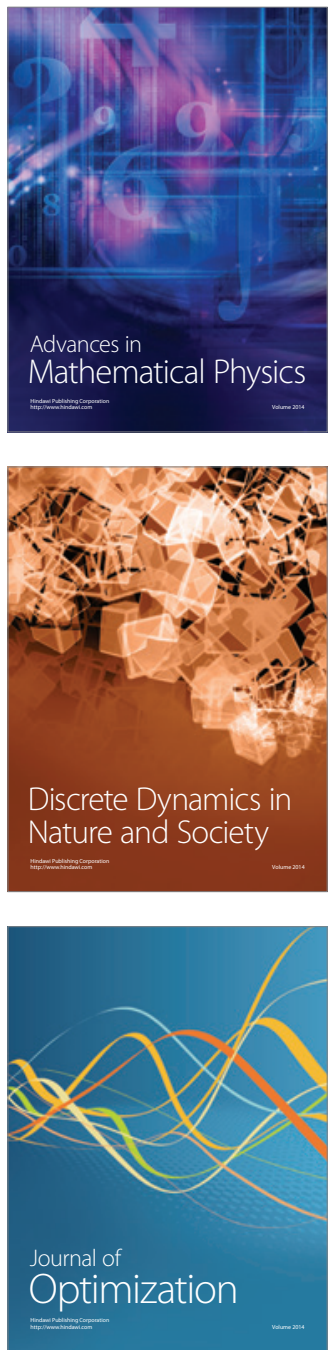